\title{
Phenomenological Model of Diffuse Global and Regional Atrophy Using Finite-Element Methods
}

\author{
Oscar Camara, Martin Schweiger, Rachael I. Scahill, William R. Crum, Beatrix I. Sneller, \\ Julia A. Schnabel, Member, IEEE, Gerard R. Ridgway, David M. Cash, Derek L. G. Hill*, Member, IEEE, and \\ Nick C. Fox
}

\begin{abstract}
The main goal of this work is the generation of ground-truth data for the validation of atrophy measurement techniques, commonly used in the study of neurodegenerative diseases such as dementia. Several techniques have been used to measure atrophy in cross-sectional and longitudinal studies, but it is extremely difficult to compare their performance since they have been applied to different patient populations. Furthermore, assessment of performance based on phantom measurements or simple scaled images overestimates these techniques' ability to capture the complexity of neurodegeneration of the human brain. We propose a method for atrophy simulation in structural magnetic resonance (MR) images based on finite-element methods. The method produces cohorts of brain images with known change that is physically and clinically plausible, providing data for objective evaluation of atrophy measurement techniques. Atrophy is simulated in different tissue compartments or in different neuroanatomical structures with a phenomenological model. This model of diffuse global and regional atrophy is based on volumetric measurements such as the brain or the hippocampus, from patients with known disease and guided by clinical knowledge of the relative pathological involvement of regions and tissues. The consequent biomechanical readjustment of structures is modelled using conventional physics-based techniques based on biomechanical tissue properties and simulating plausible tissue deformations with finite-element methods. A thermoelastic model of tissue deformation is employed, controlling the rate of progression of atrophy by means of a set of thermal coefficients, each one corresponding to a different type of tissue. Tissue characterization is performed by means of the meshing of a labelled brain atlas, creating a reference volumetric mesh that will be introduced to a finite-element solver to create the simulated deformations. Preliminary work on the simulation of acquisition artefacts is also presented. Cross-sectional and longitudinal sets of simulated
\end{abstract}

Manuscript received February 1, 2006; revised June 22, 2006. The work of O. Camara was supported in part by the EPSRC under Grant GR/S48844/01, Modelling, Understanding and Predicting Structural Brain Change. The work of W. R. Crum and M. Schweiger was supported in part by the Medical Images and Signals IRC (EPSRC GR/N14248/01 and in part by the U.K. Medical Research Council under Grant D2025/31). The work of J. A. Schnabel was supported in part by the EPSRC GR/S82503/01, Integrated Brain Image Modelling project. The work of R. I. Scahill and N. C. Fox was supported in part by the U.K. Medical Research Council under Grant G90/86 and Grant G116/143, respectively. Asterisk indicates corresponding author.

O. Camara, M. Schweiger, W. R. Crum, B. I. Sneller, J. A. Schnabel, G. R. Ridgway, and D. M. Cash are with the Centre for Medical Image Computing (CMIC), Department of Medical Physics and Bioengineering, Department of Computer Science, University College London, London WCE1 6BT, U.K. (e-mail: derek.hill@ucl.ac.uk).

R. I. Scahill and N. C. Fox are with the Dementia Research Centre, Institute of Neurology, University College London, London WC1N 3BG, U.K. (e-mail: derek.hill@ucl.ac.uk).

*D. L. G. Hill is with the Centre for Medical Image Computing (CMIC), Department of Medical Physics and Bioengineering, Department of Computer Science, University College London, London WCE1 6BT, U.K. (e-mail: derek. hill@ucl.ac.uk).

Digital Object Identifier 10.1109/TMI.2006.880588 data are shown and a visual classification protocol has been used by experts to rate real and simulated scans according to their degree of atrophy. Results confirm the potential of the proposed methodology.

Index Terms-Alzheimer's disease, atlas, atrophy simulation, creation of validation image databases, dementia, design of ground truth, finite-element methods, magnetic resonance imaging, mesh warping, modelling, validation.

\section{INTRODUCTION}

D EMENTIA, the progressive impairment of multiple cognitive domains, represents a devastating personal, socioeconomic, and public health burden that currently affects millions of elderly subjects worldwide. An estimated 24 million people have dementia today, and this figure will double in the next 20 years [1]. Symptomatic treatments for Alzheimer's disease (AD), the most common cause of dementia, are now available but have only modest benefit and are not thought to slow the relentless progression of pathology. Major efforts are under way to find effective disease-modifying agents [2]. As part of those efforts, there is great interest in using imaging in a number of different ways: to differentiate different causes of cognitive decline, to identify individuals at the earliest stages, and to evaluate the effects of pharmacological interventions on these diseases. The pattern of cerebral atrophy on magnetic resonance imaging (MRI) is currently used to help differentiate the degenerative dementias (e.g., AD from frontotemporal dementia) while the rate of atrophy may be used to detect early disease and potentially to assess disease-modifying effects of therapies [3], [4].

In early $\mathrm{AD}$, the hallmark neurofibrillary tangles and amyloid plaques are accompanied by progressive neuronal loss [5]. This loss is manifested at a macroscopic level by increased rates of cerebral atrophy, which is detectable in vivo by MRI [6]. The regional distribution of cellular damage due to different pathologies leads to different spatial patterns of atrophy in different dementias [7]-[9], varying in location, intensity, and temporal evolution. Assessment of atrophy can be confounded by volume loss due to normal aging, which also occurs in patients along with additional pathological processes [10]-[13]. Several studies indicate a strong correlation between rate of atrophy, rate of cognitive decline, and risk of future decline [3], [8], [14]-[17]. Visual inspection or manually traced measurements of regions of interest have been used previously for the quantification of atrophy. However, these methods are subject to problems of insensitivity and lack of reproducibility, as well as being labor-intensive. 
Algorithms referred to as computational anatomy [18], [19] are computerized approaches that offer automated or semi-automated solutions for MRI analysis, including quantification of cerebral atrophy. A large variety of methods have been specifically developed for cross-sectional [14], [20]-[24], [24]-[28] and longitudinal [11], [14], [29]-[36] studies. There is a lack of a gold standard against which to judge these methods or to help refine them. There is a real need for ground-truth data to be made available for validating both cross-sectional and longitudinal image analysis applications.

There have been limited attempts to model the complex effects on regional structural volumes and structural configuration that are a consequence of neurodegeneration. Cerebral atrophy has been simulated by globally scaling images [29] to effect a global reduction in brain-tissue volume. Simple scalings, however, produce an unrealistic reduction in cerebrospinal fluid (CSF) volume; in vivo CSF volumes increase as a result of tissue loss. In practice, the disease processes underlying dementia result in brain volume changes that are both tissue and location dependent. Therefore, sufficiently realistic atrophy simulation methods must be able to model local changes. Localized scaling has been used to validate atrophy measurements in small structures [21], [27], [37]. Lao et al. [27] proposed a classification method using RAVENS maps [21] and they then simulated atrophy by reducing the intensity values of these maps in a cubic region centered on a manually selected voxel, as well as by introducing morphological differences in several manually delineated structures. Chen et al. [32] simulated atrophy by replacing intensities within grey matter boundaries in a local brain region manually defined with Gaussian distributed random numbers having the CSF mean intensity and different percentages of the CSF standard deviation. Xue et al. [38] employed a technique developed by Karacali et al. [39] in which the atrophy is simulated by matching the Jacobian of a deformation, applied to the baseline scans, to the desired volumetric changes subject to smoothness and topology-preserving constraints. The main drawback of these approaches is that they do not take into account the interrelation of different tissue types.

We present a method for the simulation of atrophy in magnetic resonance (MR) images of the brain in which atrophy can be simulated in different tissue compartments and/or in different neuroanatomical structures with a phenomenological model. This model is guided by differential pathological burden in different brain regions. Once volume changes have been defined in particular structures and tissues, the overall impact on brain shape is determined by a conventional physics-based biomechanical model. This model is solved using the finite-element method (FEM) and is guided by literature values of the mechanical properties of tissue. The consequent biomechanical readjustment of structures is modelled using conventional physics-based techniques based on biomechanical tissue properties and simulating plausible tissue deformations with FEM. This validation framework was proposed by Schnabel et al. [40], for the analysis of MR mammography data. In our application, a thermoelastic model of tissue deformation is employed, controlling the rate of progression of atrophy by means of a set of thermal coefficients, each one corresponding to a different type of tissue. The thermoelastic model has no basis in the disease, but provides a straightforward way to give each element a coefficient of expansion that controls the volume change of this element relative to others.

The proposed technique can be divided into three main steps: 1) meshing of a labelled brain atlas; 2) its introduction into an FEM solver that will generate the simulated deformations; 3 ) the application of such deformations to the grey-level version of the brain atlas. In this paper, we describe the methodology and show some examples of simulations involving localized atrophy. We also show how the simulated images can be made more realistic by including realistic noise, bulk motion, and pulsatile motion artefacts. A qualitative visual classification experiment has been designed to rate the simulated and real data according to their atrophy to study whether simulated scans can mislead expert observers. Our method simulates biologically plausible degenerative changes, generating images that can be used in an objective validation of the current methods for the analysis of cross-sectional or longitudinal studies. The availability of realistic image data with a gold standard is a key aspect of improving image processing algorithms, as it is only by understanding where a technique fails that a rational approach to modifying the technique to improve its performance can be taken.

The paper is organized as follows. Section II describes the mesh generation step, while Sections III and IV are devoted to the finite-element model and the application of the resulting deformations, respectively. Some examples of simulations, including cross-sectional and longitudinal cases, are shown in Section V. In Section VI, the simulation of acquisition artefacts is described. The visual classification protocol used by experts to rate real and simulated scans and its results are detailed in Section VII. Finally, in Sections VIII and IX, we present a discussion and the conclusions of this work.

\section{MESH GENERATION}

\section{A. Reference Labelled Image}

Neuroanatomical atlases play an important role in brain analysis techniques, and are routinely used in procedures such as segmentation or spatial normalization. The most general form of an atlas is a statistical model of the population variation of anatomical structures. More common and still extremely useful are atlases which represent "typical" anatomy. They are mostly composed of manual or semiautomatic expert-segmented labels, and some are publicly available. In this work, we have combined information from two such atlases of the brain to generate the reference labelled image and mesh used by the FEM solver: the Montreal Neurological Institute (MNI) Brainweb ${ }^{1}$ [41]; and the International Consortium of Brain Mapping (ICBM) ${ }^{2}$ atlases. The MNI atlas was created from the average of 27 T1 weighted MRI acquisitions of a single young control subject, and provides a high-resolution segmentation of nine different tissue labels. The ICBM Template and Probabilistic atlases feature more specific brain parcellations, including cerebral lobes, cortical gyri, and subcortical structures.

For our purposes, we have taken into account the tissue labels from the MNI atlas, including the CSF, the grey matter (GM),

\footnotetext{
${ }^{1}$ http://www.bic.mni.mcgill.ca/brainweb/.

${ }^{2}$ http://www.loni.ucla.edu/ICBM/.
} 

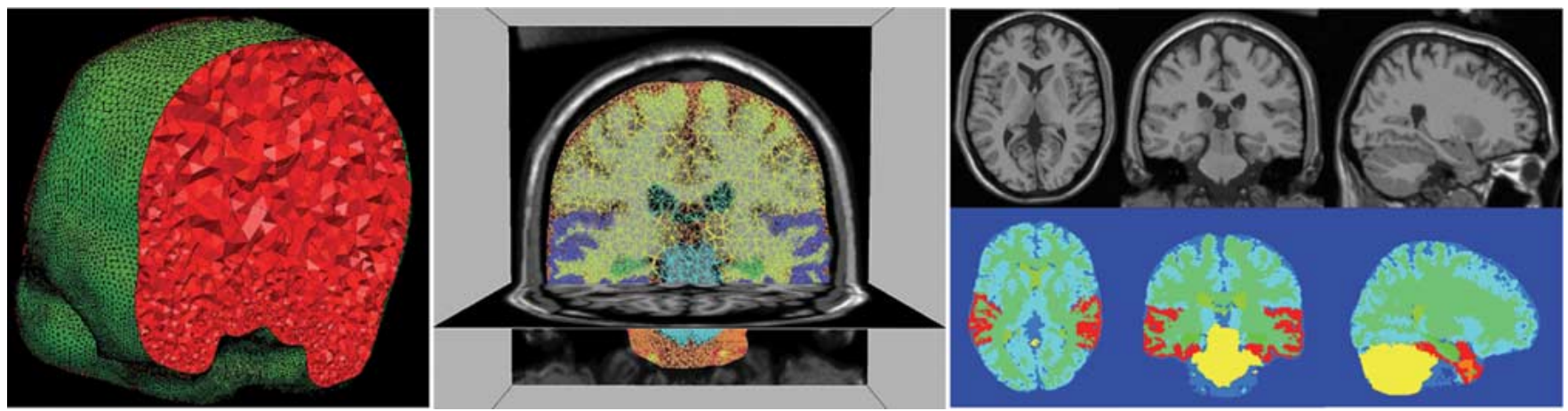

Fig. 1. Mesh generation procedure. Left: Cutting plane of the volumetric finite-element mesh. Center: Superimposition of the mesh with some slices of the greylevel version of the MNI atlas. Slices of the MNI atlas (top right) and the rasterization image (bottom right) generated from the labelled mesh, in which some of the used labels are shown (X11 colors): GM, light green; WM, cyan; cortical CSF, cornflowerblue; ventricles, greenyellow; MTL grey matter, red; subtentorial structures, yellow; hippocampi, limegreen; ERA, dark orange.

the white matter (WM), together with a zero label that includes the background, the skull, and other tissues such as the muscle or the skin. We have also incorporated labels for the hippocampi and the lateral ventricles (thus, separating the CSF into ventricular and cortical CSF labels), obtained from a semi-automatic segmentation by clinical experts [42], due to their importance as biomarkers in dementia. In addition, we have incorporated the cerebral lobe labels from the ICBM Probabilistic atlas (to generate local atrophy, e.g., in the medial temporal lobe) using an affine registration technique in order to place this atlas in the same geometrical space as the MNI one. Finally, two additional labels from the ICBM Template atlas have been included: a label covering the subtentorial structures (medulla, pons, cerebellum, brain stem, periqual grey, reticular and red nucleus, mammillary bodies) to specify boundary conditions of the FEM; and a label for the entorhinal area (ERA) because of its implication in AD. Some of these labels are illustrated in Fig. 1.

\section{B. Meshing and Labeling}

The three-dimensional (3-D) meshing of brain structures is a challenging task, especially because of the convoluted nature of the grey/CSF and white/CSF boundaries, and because partial volume effects introduce ambiguity. Our approach is to mesh the entire contents of the cranium (including CSF), and subsequently label the mesh to assign appropriate material properties to each element. This strategy allows the modelling of a different behaviour for each tissue compartment and/or anatomical structure.

Therefore, the first phase consists of the generation of a surface mesh from the intracranial surface using the classical marching cubes [43] algorithm, followed by some postprocessing steps (decimation, smoothing, and selection of biggest connected component) to guarantee the creation of a continuous surface brain mesh. A volume (3-D) finite-element (four-noded tetrahedra) mesh was then obtained from the intracranial mesh surface using the NETGEN ${ }^{3}$ openSource software [44]. The final step is the labelling of the 3-D mesh using the labelled voxel atlas.

NETGEN is based on the combination of the Delaunay algorithm with a rule-based advancing front method. It also allows

\footnotetext{
${ }^{3}$ http://www.hpfem.jku.at/netgen/
}

TABLE I

Elastic Material Properties (Young's Modulus $E \times 10^{3} \mathrm{NM}^{-2}$ AND POISSON'S RATIO $\nu$ ) AND NUMBER OF MESH ElEMENTS OF EACH Compartment. MTL: Medial Temporal Lobe

\begin{tabular}{|c|c|c|c|}
\hline region & $E$ & $\nu$ & $\sharp$ and \% of elements \\
\hline \hline Cortical CSF & 0.1 & 0.05 & $301280(34.69 \%)$ \\
\hline Ventricles & 0.1 & 0.05 & $1149(0.13 \%)$ \\
\hline Grey Matter & 23.2 & 0.45 & $161700(18.62 \%)$ \\
\hline White Matter & 34.8 & 0.45 & $108169(12.46 \%)$ \\
\hline Hippocampi & 29.0 & 0.45 & $2685(0.31 \%)$ \\
\hline Subtentorial & 29.0 & 0.45 & $92103(10.61 \%)$ \\
\hline Entorhinal Area & 29.0 & 0.45 & $4033(0.47 \%)$ \\
\hline MTL Grey Matter & 23.2 & 0.45 & $110119(12.68 \%)$ \\
\hline
\end{tabular}

adaptive meshing, generating elements with different sizes depending on their distance to surfaces of high curvature, which is desirable in our application since high precision is required in the cortex. We generated a tetrahedral mesh with very fine resolution (163765 nodes and 868404 elements, with 61132 surface elements), which allows a high number of elements for the most critical structures or regions such as the ERA, the hippocampi, or the interface between the grey and white matter. The number of tetrahedra corresponding to each tissue label is listed in Table I ${ }^{4}$. The geometrical quality of the elements of the mesh is critical for the convergence and stability of the FEM solution, as poor quality elements can lead to poorly conditioned stiffness matrices [45]. We have computed a widely used criterion of element quality that is defined as the ratio between the radius of the inscribed circle $r_{\text {in }}$ and the longest edge $l_{\max }$ in the tetrahedron

$$
Q=2 \sqrt{6} \frac{r_{\text {in }}}{l_{\max }} .
$$

This criterion is negative in folded elements and equal to 1 in case of optimal quality, i.e., an equilateral tetrahedron. In Section VII-B, we will use this criterion to assess the quality of the generated mesh, shown in Fig. 1, and some warped meshes.

\footnotetext{
${ }^{4}$ The remaining tetrahedra were assigned to the background class
} 
The obtained values of $Q$ assure good behavior of the FEM solver when using the generated volumetric mesh.

The mesh labelling step assigns a set of particular biomechanical properties to each tetrahedron. Label information is mapped from the reference labelled image into the mesh through a rasterization procedure [46]. This computes the whole set of voxels that intersects with each mesh element, and then computes the percentage/probability of each label for each tetrahedron within the intersection set; the most common tissue type is assigned to the element. Fig. 1 shows some slices of the grey-level version of the MNI atlas and the corresponding rasterized image obtained from the volumetric mesh. In order to reduce the impact of the discretization of the mesh, we apply a partial volume tissue model to each tetrahedron in the FEM solver, as detailed in Section III-C.

\section{Finite-Element Deformation Model}

The use of FEM for biomechanical modelling is an active field of research, being a valuable tool in clinical applications such as image-guided surgery. It offers the possibility of predicting mechanical or physical deformations through the simulation of anatomical structures and their interactions, as well as the estimation of material properties from observations. FEM have been applied in the modelling of different anatomical structures, involving liver [47]-[49], heart [46], [50], or mammography data [40], [51]-[54]. With respect to brain modelling, FEM have been used for image-guided surgery [55]-[58] or interventional [59], [60] procedures, registration systems [61], tumor growth simulation [62], or the estimation of brain tissue biomechanical properties [63], amongst other applications.

In the following sections, we present the FEM model we have developed, as well as the definition of the boundary conditions and the biomechanical tissue properties. Finally, we describe the method for the estimation of the thermal coefficients that will guide the FEM solver.

\section{A. Model}

We use a thermoelastic model of soft tissue deformation to simulate the change in brain structure caused by degenerative diseases. The deformation model employs a linear elastic finiteelement method based on the TOAST package [64], which is freely available. 5 The implementation of the FEM model follows a standard approach (see, for example, [65]), whereby each tetrahedral mesh element is assigned a set of elastic material properties represented by an elasticity matrix $\mathrm{D}$. In the case of isotropic elastic deformations, $D$ is symmetric and can be expressed in terms of two parameters, usually given as Young's modulus $E$ and Poisson's ratio $\nu$, or equivalently by the Lamé modulus $\lambda$ and shear modulus $\mu$, defined as

$$
\lambda=\frac{\nu E}{(1+\nu)(1-2 \nu)}, \quad \mu=\frac{E}{2(1+\nu)} .
$$

The elastic coefficients are assumed time-invariant. The deformation of the mesh is induced by assigning an isotropic thermal expansion coefficient $\alpha^{(i)}$ to each element $i$, and simulating a global temperature change $\Delta T$. The resulting isotropic

\footnotetext{
${ }^{5}$ http://www.medphys.ucl.ac.uk/martins/toast/index.html
}

thermal expansion enters the description of elastic deformation in the form of an initial element strain

$$
\varepsilon_{0}^{(i)}=\left\{\alpha^{(i)} \Delta T, \alpha^{(i)} \Delta T, \alpha^{(i)} \Delta T, 0,0,0\right\}
$$

where the relation between stresses $\sigma$ and strains $\varepsilon$ is given by

$$
\sigma^{(i)}=\mathrm{D}^{(i)}\left(\varepsilon^{(i)}-\varepsilon_{0}^{(i)}\right)+\sigma_{0}^{(i)} .
$$

Assembling all element contributions of the mesh leads to the linear system

$$
\mathrm{Ku}+\mathbf{f}+\mathbf{f}^{\prime}=\mathbf{r}, \quad K_{i j}=\int_{V} \mathrm{~B}_{i}^{T} \mathrm{DB}_{j} d V
$$

with stiffness matrix $\mathrm{K}$. In an $n$-noded element, $\mathrm{B}$ is a $6 \times 3 n$ strain displacement matrix $B=\left\{B_{i}\right\}$, and

$$
\mathbf{f}^{\prime}=\int_{V} \mathrm{~B}^{T} \mathrm{D} \varepsilon_{0} d V
$$

contains the volume forces arising from the initial thermal strain, $\mathbf{f}$ combines all other surface and volume force terms, $\mathbf{r}$ defines explicit displacements, and $\mathbf{u}$ is the vector of nodal displacements.

\section{B. Boundary Conditions and Mechanical Properties of Brain Tissue}

The surface of the mesh was assumed to coincide with the inner surface of the skull, and homogeneous Dirichlet boundary conditions were introduced using a Payne-Irons ("big spring") method to suppress the displacements of boundary nodes. The same strategy was applied to the mesh nodes corresponding to the subtentorial structures since atrophy induced by dementia is in general localized in the supratentorial area.

There is not a consensus on the optimal Young's modulus $E$ and Poisson's ratio $\nu$ of the different brain tissues, as demonstrated in the reviews of Kruse et al. [66] and Hagemann et al. [61]. Nevertheless, the choice of the elastic properties is not critical in the model presented here, because the boundary conditions enforce a constant global volume, while the relative changes in tissue region volumes are driven by the ratio of thermal coefficients. Furthermore, the mechanical properties of brain tissue are directly relevant to how the brain responds to an external force but are much less relevant to the response of the brain to diffuse cell damage and death that results in active structural readjustment. Therefore, we have adopted the shear modulus values proposed by McCracken et al. [67], which have been estimated using magnetic resonance elastography imaging: $12 \mathrm{kPa}$ for white matter and $8 \mathrm{kPa}$ for grey matter. For the Poisson's ratio, we have chosen a value of 0.45 for each brain tissue. Even if the human brain is expected to be more incompressible than that, it has been shown that the choice of an appropriate set of boundary conditions is more critical than the exact value of the material properties [68]. Using (2) and the values of the Poisson's ratio and the shear modulus stated above, we obtain a Young's modulus of $34.8 \mathrm{kPa}$ for the white matter and $23.2 \mathrm{kPa}$ for the grey matter. If we consider that the 

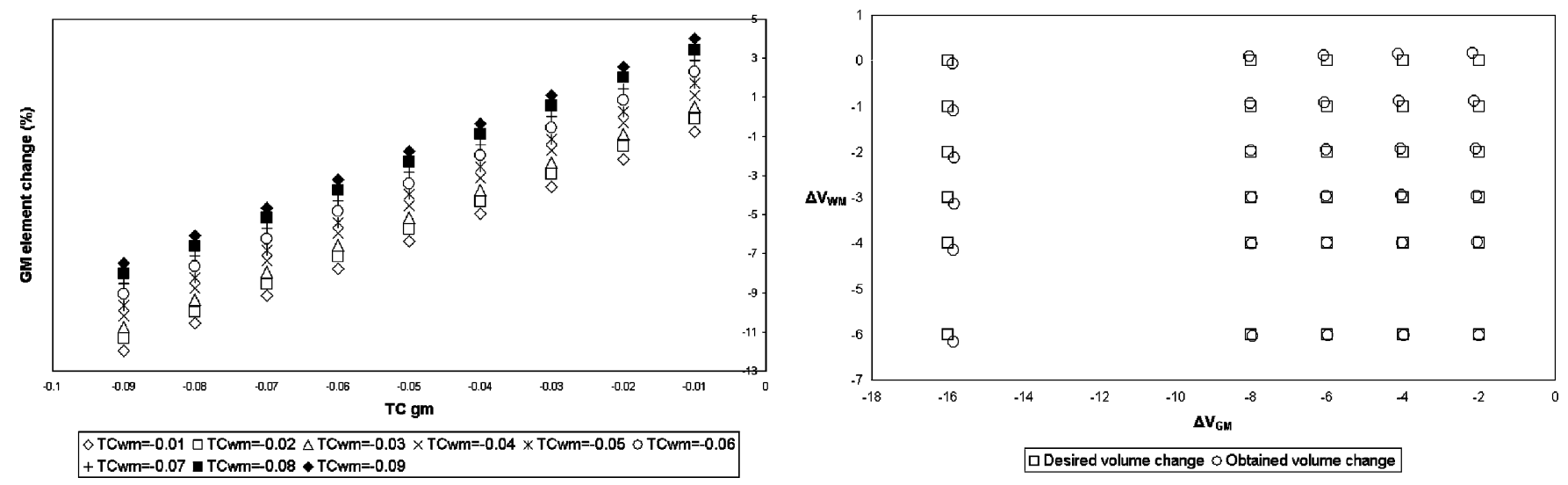

Fig. 2. Relation between thermal coefficients and desired volume change. Left: Graph representing the percentage of GM element change when varying the thermal coefficients of the GM and WM (TCgm and TCwm, respectively). Right: Graph illustrating the good agreement between the desired and the obtained volume change of GM and WM.

hippocampi, the entorhinal area, and the subtentorial structures are composed of an equal percentage of grey and white matter, then their material properties are the average of the ones corresponding to the grey and white matter $(E=29.0 \mathrm{kPa})$. With respect to the CSF, we assume it to be a very soft and compressible tissue, with low Young's modulus and Poisson's ratio. All the values used in this work are summarized in Table I.

\section{Thermal Coefficient Computation}

Given a segmentation of domain $\Omega$ into $N$ tissue types or anatomical structures $\Omega_{j}, \Omega=\cup_{j} \Omega_{j}, j=1, \ldots, N$, we assume the thermal coefficient $\alpha$ to be constant within each region $\Omega_{j}$, so that $\alpha$ is expressed in a piecewise constant basis, $\alpha(\mathbf{r})=\alpha_{j}$, $\mathbf{r} \in \Omega_{j}$. Once the material properties have been defined, we need to estimate the thermal coefficients $\alpha_{j}$ that will provide a mesh deformation consistent with the desired volume change that we want to apply to each tissue segment $\Omega_{j}$. These thermal coefficients will generate a deformation field that will be applied to the original mesh, generating a displaced mesh. Subsequently, we integrate the differences in element volumes between the original and displaced meshes over each region $\Omega_{j}$ in order to compute the obtained volume change. This obtained volume change will provide the ground-truth information needed for validation.

Initially, the relation between the $\alpha_{j}$ and volume changes $\Delta V_{j}$ was obtained empirically, i.e., by running the FEM solver a sufficient number of times with different values of $\alpha_{j}$ to fit a linear model to the resulting volume changes. For instance, if we want to explicitly change the volumes of the grey and white matter, their corresponding values of $\alpha_{j}$ will be estimated, given a volume change, $\Delta V_{\mathrm{GM}}$ and $\Delta V_{\mathrm{WM}}$, by the following equations:

$$
\begin{aligned}
\Delta V_{\mathrm{GM}} & =m_{1} \alpha_{\mathrm{GM}}+m_{2} \alpha_{\mathrm{WM}}+m_{3} \\
\Delta V_{\mathrm{WM}} & =n_{1} \alpha_{\mathrm{GM}}+n_{2} \alpha_{\mathrm{WM}}+n_{3}
\end{aligned}
$$

where the $m$ and the $n$ parameters will be estimated by the fitting procedure. The assumption of linearity has been shown to be appropriate, as can be seen in Fig. 2. In this figure, we also show the differences between the desired and the obtained volume change for a set of 30 simulations (mean difference of $0.35 \%$ \pm 0.41 and $0.14 \% \pm 0.08$ for GM and WM, respectively) confirming the appropriateness of the linear model.

As pointed out in Section II-B, we may make use of the information provided by the rasterization approach about the percentages of each tissue on each mesh element in order to reduce the effect of the mesh discretization. Hence, for each mesh element, $i$ the thermal coefficient $\alpha^{(i)}$ will be a partial volumeweighted average of the region thermal coefficients contributing to the element

$$
\alpha^{(i)}=\sum_{j} p_{j}^{(i)} \alpha_{j}
$$

where $p_{j}^{(i)}$ is the fractional volume contribution of region $j$ to element $i$.

This method of recovering the thermal coefficients for a given set of target region volume changes can be generalized for an arbitrary number of regions by applying a linear inversion technique. The relationship between changes of $\alpha_{j}$ and $\Delta V_{i}$ in regions $i$ and $j$ is expressed by the Jacobian matrix

$$
\mathrm{J}_{i j}=\left[\frac{\partial V_{i}}{\partial \alpha_{j}}\right]
$$

which we compute with a finite difference scheme by explicitly perturbing each $\alpha_{j}$. Now we form the linear system

$$
\mathrm{J}[\alpha]=[\Delta V] .
$$

Note that due to the boundary conditions, the volume changes on the right-hand side of (10) are not independent, because $\sum_{i} \Delta V_{i}=0$ is required. Likewise, the thermal coefficients on the left are only determined up to an arbitrary additive term, which can be taken into account by fixing one of the coefficients, e.g., $\alpha_{0}=0$. The resulting linear system is, therefore, quadratic of dimension $N-1 \times N-1$ and can be solved by a standard LU decomposition. 


\section{WARPING}

The FEM solution has produced a set of displacement vectors $d=(d x, d y, d z)$ defined at each node of the volumetric mesh. In order to simulate atrophy in the atlas image, we need to use interpolation techniques to generate a displacement field at each voxel of the image. For doing so, we can weight the displacement vectors $d$ of the nodes in the element containing a given voxel $x$ by the element linear shape function $S(j)$ [40], [69]

$$
d(x)=\sum_{j=1}^{4} S(j) d_{j}
$$

Using the FEM shape interpolation defined in (11) above, a dense voxel displacement within the volumetric mesh can be calculated, and the new image intensities can be interpolated using a truncated sinc interpolation kernel [70]. We have adopted a different interpolation strategy outside the mesh, where no deformation information is available from the FEM model, and at mesh boundaries in order to avoid intensity discontinuities due to decimation and smoothing steps in the mesh generation stage. Here, we employ a scattered data interpolation technique [71] based on multilevel $B$-splines, in order to blend the FEM shape interpolated displacements within the mesh and at the mesh boundary with smooth, cubic B-spline interpolants at outside locations. Five hierarchical levels of B-splines with a decreasing grid spacing were used (20, 10, 5, 2.5, and $1.25 \mathrm{~mm})$.

Using such an interpolation scheme, we can warp the greylevel version of the MNI atlas without noise using the dense displacement field generated by the FEM solver. Finally, we add Rician distributed noise (with an amplitude equal to $2 \%$ of the maximum intensity value of the image) to the atrophy simulated image.

\section{EXAMPLES OF SimUlations}

The proposed methodology has been designed to make it possible to simulate many neurodegenerative diseases. The illustrative examples shown in this section are focused on $\mathrm{AD}$, as it is the most common cause of dementia. In AD, the early and most severely affected structures are in the medial temporal lobe, such as the entorhinal cortex (ERC) and the hippocampus [25], [72]-[79], followed by paralimbic cortical areas and reaching the neocortex at the later stages the disease [5], [25], [80]-[82]. From this topographic progression of the pathology, we expect a strong correlation of the regional measures based on hippocampus and ERC with the conversion from normal to mild cognitive impairment (MCI) and global measures of hemispheric atrophy rates, whole brain and ventricle (and temporal horn) strongly associated with clinical progression at later stages of the disease [83]. Therefore, medial temporal structures seem an obvious choice for biomarkers due to their early pathologic involvement in the AD process, but changes in whole-brain and ventricular volume can give complementary data by providing a summation of the widely distributed cerebral volume loss that are ongoing by the time an individual is even mildly affected [83]-[85].

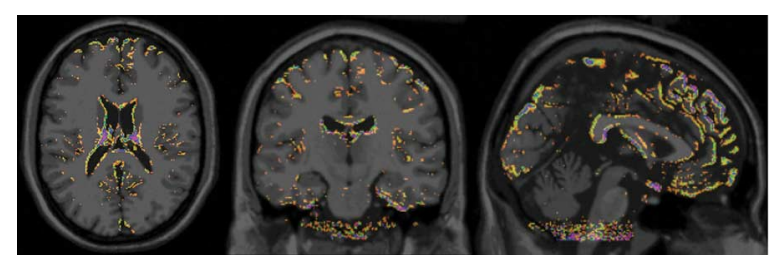

Fig. 3. Results of the cross-sectional simulation example. Superimposition of the MNI atlas with results obtained from the mass-univariate voxel-wise twosample $t$-test.

Based on these conclusions, we have used the proposed methodology to generate two sets of simulated data. As pointed out above, the main goal of this work is the generation of ground-truth data for the validation of the existing atrophy measurement methods. In Section I, we classified such techniques into cross-sectional and longitudinal designed ones. Hence, we present one illustrative example for each of these categories.

\section{A. Cross-Sectional Simulation}

The cross-sectional simulation consisted of the generation of two cohorts of subjects from the MNI-ICBM atlas mesh. The first one represents a group of 10 controls, in which the FEM solver induces an average reduction in the brain of $0.49 \%$ $( \pm 0.02)$. Accordingly, the second cohort is composed of $10 \mathrm{AD}$ patients, in which an average reduction in the brain of $2.21 \%$ $( \pm 0.11)$ is applied. These values are inspired by the review of MR-based measures of atrophy in Preboske et al. [86].

We have applied the SIENAX [28] technique to these two groups of subjects, using the freely available software, ${ }^{6}$ developed at the FMRIB Analysis Group, Department of Clinical Neurology, Oxford, U.K. We have used the default parameters of SIENAX and the study aimed to detect differences in brain tissue between the two simulated cohorts. SIENAX results on brain volume change are quite similar to the ground truth ones, knowing that this technique has an estimated $0.5 \%-1 \%$ brain volume accuracy [28]: an average reduction of $0.62 \%( \pm 0.05)$ for controls, and an average reduction of $2.32 \%( \pm 0.1)$ for patients.

Since the cohorts are already spatially normalized, the differences between them can be seen clearly in an image formed by subtracting one group's mean image from the other's. To investigate the significance of these differences, we performed a mass-univariate voxel-wise two-sample $t$-test. Fig. 3 shows the superimposition of the MNI atlas image with the negative base-10 logarithm of each voxel's $p$-value from this test. As expected, the most significant values are located around the lateral ventricles and the cortical GM.

\section{B. Longitudinal Simulation}

Besides the quantification of atrophy, it is also critical to identify the spatial pattern of the disease, especially to distinguish between different types of dementia. Therefore, the longitudinal example is based on the generation of a set of simulated images approximately mimicking the distribution of regional change in

\footnotetext{
${ }^{6}$ http://www.fmrib.ox.ac.uk/fsl/
} 
TABLE II

Volume Changes (\% With Respect to THE Baseline) Simulated in THE LONGITUdinal Example. MTL: Medial Temporal LoBe. Most SigNIFICANT VOLUME CHANGES IN THE AD PATIENTS IN EACH TIMEPOINT ARE MARKED IN BOLD

\begin{tabular}{|c||c|c|c||c|c|c||}
\hline \multicolumn{1}{|c||}{\multirow{2}{*}{ Structures }} & \multicolumn{3}{c||}{ Normal aging } & \multicolumn{3}{c||}{ Alzheimer's disease } \\
\cline { 2 - 7 } & $\mathrm{t} 1$ & $\mathrm{t} 2$ & $\mathrm{t} 3$ & $\mathrm{t} 1$ & $\mathrm{t} 2$ & $\mathrm{t} 3$ \\
\hline \hline Cortical CSF & 1.90 & 3.76 & 5.66 & 1.98 & 5.27 & $\mathbf{1 6 . 8 2}$ \\
\hline Ventricles & 2.18 & 4.31 & 6.49 & 2.23 & $\mathbf{5 . 8 2}$ & $\mathbf{1 9 . 3 4}$ \\
\hline Grey Matter & -0.65 & -1.28 & -1.92 & -0.65 & -1.30 & $\mathbf{- 5 . 3 1}$ \\
\hline White Matter & -0.17 & -0.32 & -0.49 & -0.16 & -0.32 & $\mathbf{- 1 . 3 3}$ \\
\hline Hippocampi & -1.40 & -2.79 & -4.21 & $\mathbf{- 3 . 3 3}$ & $\mathbf{- 6 . 3 9}$ & $\mathbf{- 9 . 3 1}$ \\
\hline Subtentorial & 0.02 & 0.03 & 0.05 & 0.02 & 0.04 & 0.13 \\
\hline Entorhinal Area & -2.91 & -5.80 & -8.67 & $\mathbf{- 5 . 1 1}$ & $\mathbf{- 9 . 6 0}$ & $\mathbf{- 1 3 . 6 8}$ \\
\hline MTL Grey Matter & -0.63 & -1.28 & -1.92 & -0.65 & $\mathbf{- 4 . 6 8}$ & $\mathbf{- 8 . 5 9}$ \\
\hline Whole Brain & -0.36 & -0.71 & -1.08 & -0.38 & -1.01 & $\mathbf{- 3 . 2 0}$ \\
\hline
\end{tabular}

$\mathrm{AD}$ according to the severity of the disease. Three different atrophy simulated images are generated: in the first one $(t 1)$, only changes in the hippocampi and the entorhinal area are applied; the second image $(t 2)$ also includes grey matter changes in the medial temporal lobe and an expansion of the lateral ventricles; finally, a global reduction of the whole brain is applied to generate the third simulated image $(t 3)$. In addition, we have generated a corresponding set of simulated images at the three timepoints representing volume change induced by normal aging. We chose a range of values (see Table II) which included a range of cerebral volume losses, as a percentage of baseline, that is consistent with published rates of atrophy and study durations [83], [87] of 6-24 months. Fig. 4 shows the difference images between the normal aging and the $\mathrm{AD}$ simulated images at each timepoint, demonstrating the potential of the proposed methodology.

\section{Simulation of ACQUisition ARTEFACTS}

The presence of artefacts in the MR scans due to imperfections during the acquisition procedure may have a strong influence on the performance of the atrophy measurement techniques. Therefore, we have developed methods to simulate acquisition artefacts, making the generated ground-truth data more realistic. In this work, we deal with two of the most common causes of acquisition artefacts, a rotational motion during the scan, and a pulsatile motion.

\section{A. Rotational Motion}

Rotational motion during a scan causes inconsistencies in the $k$-space signal that result in blurring and ghosting in the image domain. The method we developed to simulate rotational motion was as follows.

A motion-free 3-D image volume was converted into a complex image by treating the modulus image as the real part, and setting the imaginary component to zero. This complex image was then rotated by $5^{\circ}$ about an axis through the internal auditory meatus, simulating a slight nodding of the patient during the scan. Bicubic interpolation was used in the rotation, and voxels entering the volume were assigned the value zero. The original and rotated images were then both Fourier-transformed into $k$-space. A motion-corrupted $k$-space was subsequently generated by assembling portions of the $k$-spaces corresponding to the unrotated and rotated images. The motion-corrupted $k$-space was then transformed back to the image domain to give the motion-corrupted volume. The $k$-space samples were combined as sagittal planes representing a sagittal MR acquisition orientation.

The appearance of the motion artefacts is influenced by which region (its distance to the centre of the $k$-space) of $k$-space is being sampled as the motion takes place, and whether there is a single step motion or a periodic motion. Our technique allows complete freedom in the selection of the time of the motion in the scan, and for the examples in this paper, we simulated step motion by combining the first $57 \%$ of the unrotated $k$-space, with $43 \%$ of the rotated $k$-space. We simulated the motion just after half way through the acquisition as the artefact is more noticeable the nearer to the centre of $k$-space it occurs. Periodic motion, representing a repeated nodding of the head, was simulated by generating the motion-corrupted $k$-space from portions of the unrotated and rotated $k$-space, alternating between the two at $44 \%$, $48 \%, 52 \%$, and $56 \%$. The motion-corrupted $k$-space was transformed back to the image domain, and a zero-mean with standard deviation of five Gaussian noise was added independently to the real and imaginary components before generating modulus image for subsequent analysis. The top row of Fig. 5 shows an example of simulated periodic rotational motion.

\section{B. Pulsatile Motion}

Another important artefact in MR images is pulsatile motion, which typically leads to streaks across the image in the slow-phase encoding direction. These artefacts arise from major blood vessels in the field of view, such as the carotid artery where it passes through the carotid siphon, and result from a signal in the vessel that varies from shot to shot during the acquisition. Simulated pulsatile motion artefacts for array coils were added to the simulated data as follows.

The 3-D modulus images were converted to a complex image as described above, and four different coil views were obtained by multiplying the image by four separate coil sensitivity profiles. Because the pulsatility artefact is predominantly in the slow-phase encode direction, each plane of the $k$-space was treated as being acquired instantaneously (contrast being largely determined by the acquisition of the center portion of $k$-space) and $1.73 \mathrm{~s}$ apart from the next. A cylinder corresponding to the carotid artery was manually delineated from the image, and the intensity of this modulated according to a literature-based model of the flow in the carotid artery [88]. For each shot in the slow-phase encode direction, a different image was simulated by modulating the intensity of the carotid signal in the delineated cylinder. These images were transformed into the $k$-space, and one $k$-space plane from each image was combined to form a pulsatile-motion corrupted $k$-space based on an estimated heart rate of $60 \mathrm{bpm}$. Because this is a one-dimensional motion, it was implemented just for the lines containing the carotid, rather than the entire image, in order to reduce computational cost. The resulting image, as can be seen in Fig. 5, has a simulated flow artefact running right to left. 


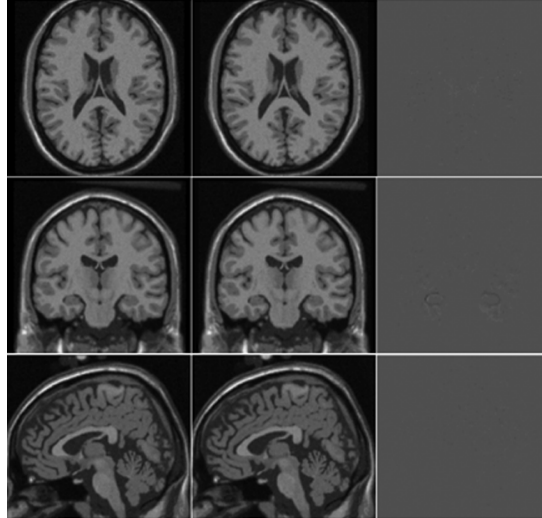

(a)

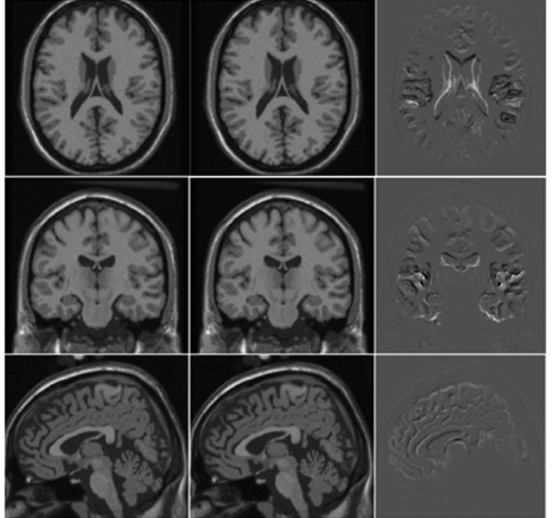

(b)

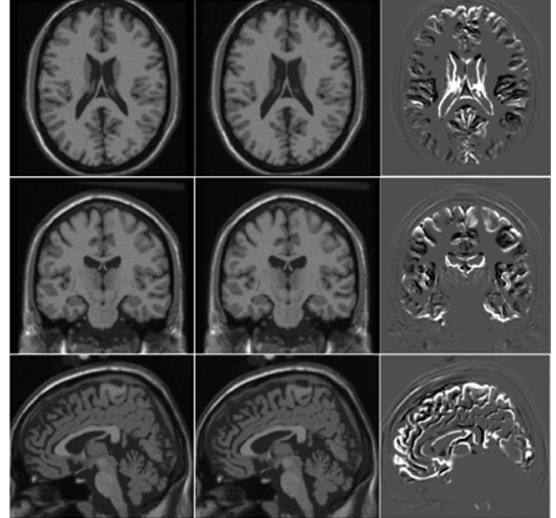

(c)

Fig. 4. Results of the longitudinal simulation example. Axial (top), coronal (middle), and sagittal (bottom) slices of the normal aging, AD simulated, and their difference (left, center, and right in each subset of three images, respectively) images at each timepoint. (a) $t 1$. (b) $t 2$. (c) $t 3$.
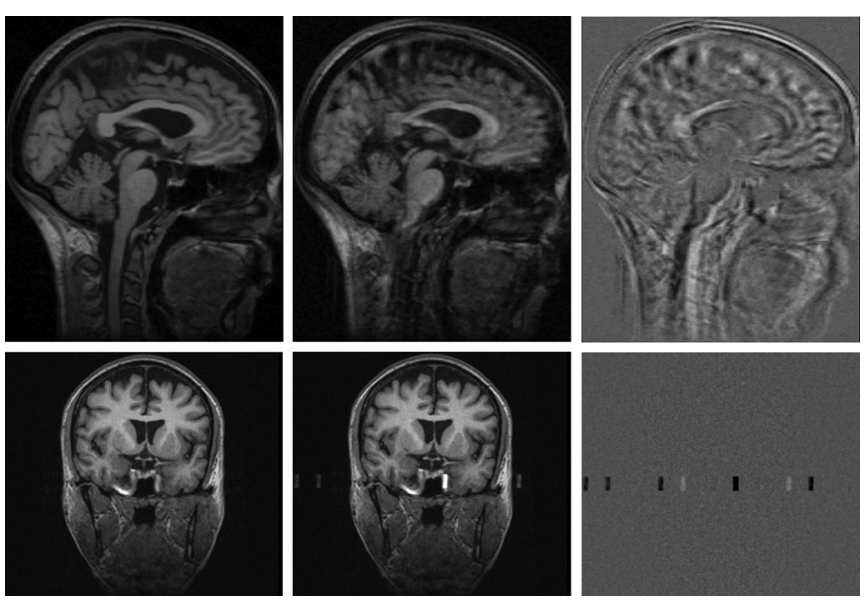

Fig. 5. Simulation of acquisition artefacts. Top: Original atrophy simulated image (left), simulation of periodic rotational motion (center), and the difference image (right) between them. Bottom: Atrophy simulated image with array coil noise (left), simulation of pulsatile motion (center), and the difference image (right) between them.

\section{Visual Classification PROTOCOL}

The evaluation of the degree of realism of a simulated image is quite a challenging task in our application. However, an experiment was designed in which a visual classification protocol was used by experts to rate real and simulated MRI data of controls and patients with atrophy. We could then study whether experts consistently rate real and simulated data in a different way. The experiment used two matched cohorts of 20 controls and 20 mild AD subjects, together with nine atrophy simulated images. A mesh warping technique was employed to put the volumetric mesh presented in Section II into the anatomical space of nine of the controls, thus generating nine control-specific meshes. Thereafter, they were introduced into the FEM solver, together with volumetric difference estimates (whole brain, hippocampus, lateral ventricles) between both cohorts that have been previously computed using manual tracing techniques. The output of the FEM solver was a set of deformations that were applied to the control images to generate a group of atrophy simulated images equivalent to the cohort of mild AD subjects. The
TABLE III

DEMOGRAPHICS OF THE SUBJECTS INVOLVED IN THE VISUAL ClassifiCATION Protocol. MEAN \pm STD. M: MALES; F: FEMALES

\begin{tabular}{|c||c|c|c|c|}
\hline & $\begin{array}{c}\text { Age } \\
\text { (years) }\end{array}$ & Gender & $\begin{array}{c}\text { Brain volume } \\
\text { volume }(\mathrm{ml})\end{array}$ & $\begin{array}{c}\text { Hippocampal } \\
\text { volume }(\mathrm{ml})\end{array}$ \\
\hline \hline Controls & $69.5 \pm 6.3$ & $10 \mathrm{M} / 10 \mathrm{~F}$ & $1110 \pm 120$ & $2.59 \pm 0.27$ \\
\hline $\mathrm{AD}$ & $68.4 \pm 6.4$ & $10 \mathrm{M} / 10 \mathrm{~F}$ & $975 \pm 68$ & $2.14 \pm 0.43$ \\
\hline
\end{tabular}

three sets of images, including real and simulated data, were then introduced to the visual protocol.

\section{A. Database Characteristics, Image Acquisition, and Preprocessing}

Twenty healthy elderly controls and 20 individuals with a diagnosis of probable AD [89] were included in the study (see Table III). These subjects were enrolled in a longitudinal research project at the Dementia Research Centre, Institute of Neurology, University College London, U.K., and underwent serial MRI, clinical, and neuropsychological assessment over a period of at least one year. A subset of nine controls (matched with the full group for age, gender, and brain and hippocampal volumes) was selected for the simulated atrophy model.

T1-weighted volumetric MR images were acquired on a 1.5 Tesla Signa unit (General Electric, Milwaukee) using a $256 \times$ 256 matrix to provide 124 contiguous $1.5-\mathrm{mm}$ coronal slices through the head (acquisition parameters: time to repeat, $15 \mathrm{~ms}$; time to echo, $5.4 \mathrm{~ms}$; flip angle $15^{\circ}$; field of view $24 \times 24 \mathrm{~cm}$ ). Whole brain and hippocampal segmentations were performed on all scans using the MIDAS software [42] according to previously described protocols [90]. Volumes are given in Table III.

\section{B. Mesh Warping}

Mesh-warping (MW) [91], [92] (sometimes referred to as mesh-matching [93]) is a generic technology for individualizing a reference mesh to a new subject using image registration. It can be divided into three main steps: 1) reference mesh generation; 2) image registration; 3) mesh transformation. Here, the aim is to transform the MNI-ICBM atlas mesh to match an individual brain. Therefore, the registration step must compute the 
TABLE IV

FitTing AND GEOMETRIC (PERCENT) QUALity. RO: RegistRation OVERLAPS; MW: MESh-WARPING

\begin{tabular}{|c||c|c||c|c|c|c|}
\hline \multirow{2}{*}{ Registration } & \multicolumn{3}{|c||}{ Fitting } & \multicolumn{4}{c|}{ Geometrical quality $(Q(\%))$} \\
\cline { 2 - 7 } & RO & MW & $<0$ & $0-0.3$ & $0.3-0.7$ & $>0.7$ \\
\hline \hline Original & 0.97 & 0.93 & 0.00 & 0.00 & 26.48 & 73.52 \\
\hline Affine & $0.76 \pm 0.03$ & $0.75 \pm 0.02$ & $0.00 \pm 0.00$ & $0.00 \pm 0.00$ & $28.78 \pm 1.03$ & $71.22 \pm 1.03$ \\
\hline Fluid & $0.89 \pm 0.01$ & $0.83 \pm 0.03$ & $0.00 \pm 0.00$ & $0.31 \pm 0.16$ & $61.77 \pm 2.36$ & $37.92 \pm 2.45$ \\
\hline \hline
\end{tabular}
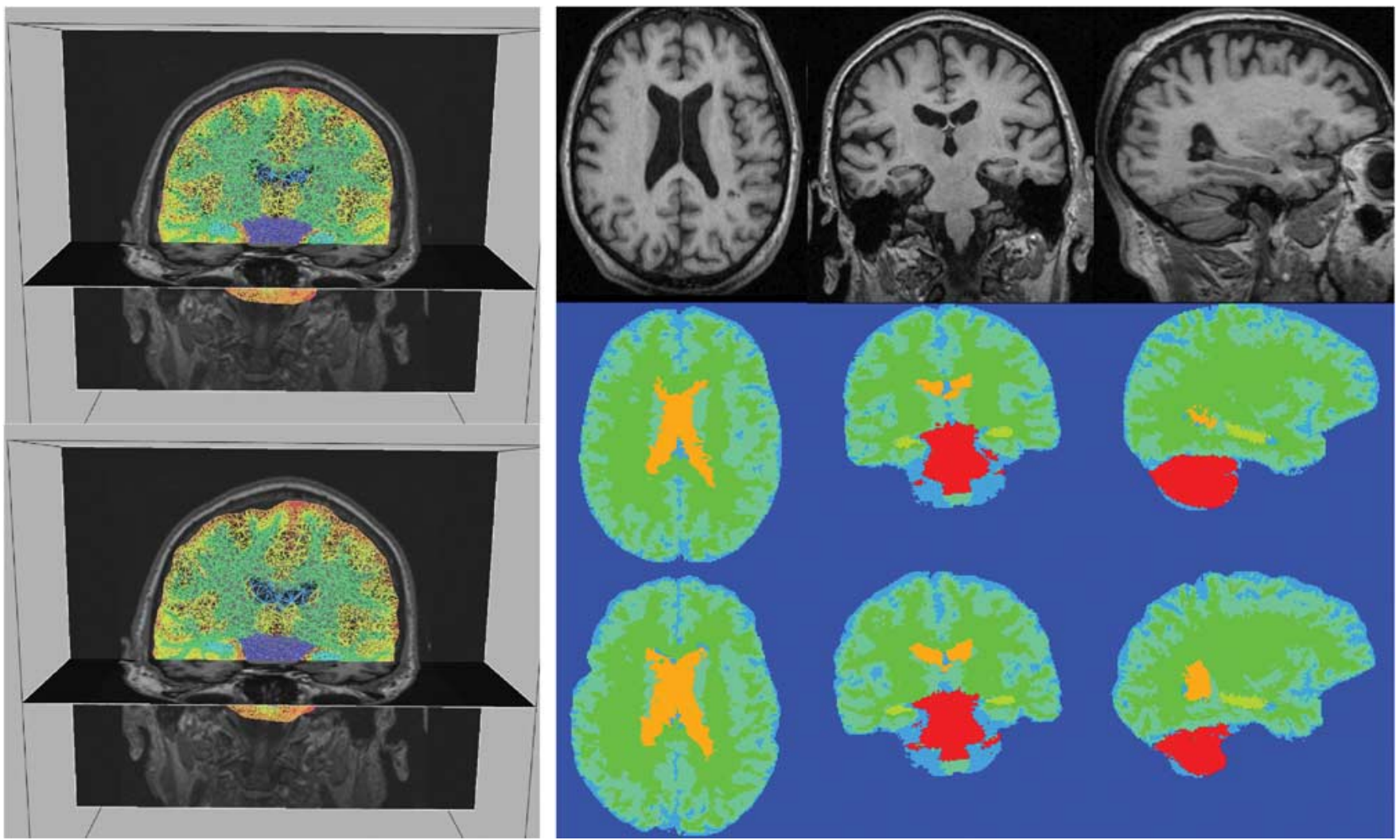

Fig. 6. Mesh warping procedure. Left: Superimposition of the mesh with some slices of the grey-level version of the MNI atlas after affine (top) and fluid (bottom) MW. Right: Slices of the control grey-level image (top) and the rasterization image generated from the labelled mesh after affine (middle) and fluid (bottom) MW.

optimal geometrical transformation between each control image and the grey-level version of the atlas.

First, each grey-level image of the controls was registered to the atlas using a 12 degrees of freedom affine registration [94] with normalized mutual information as the image similarity measure. For MW, it is essential to avoid folding in the deformation fields (nondiffeomorphic transformations) applied to the meshes, in order to preserve the geometrical quality of their elements and ensure the convergence and numerical stability of subsequent finite element analysis. In consequence, we applied a fluid registration technique [95] to determine a diffeomorphic transformation. The resulting transformation was applied to the MNI-ICBM atlas mesh, creating a set of meshes specific to the control subjects.

Geometric and fitting quality of the warped meshes were used to evaluate the MW procedure. For the geometric quality, we used the criterion $Q$ described in Section II-B. For the fitting quality, first, a simple check of the registration quality was performed by computing the classical Jaccard overlap measure [96] of semiautomatically obtained brain-masks before and after each registration stage. Overlap values (mean \pm STD) are shown in Table IV. We have also computed the overlaps between the brain-masks and the rasterization (see Section II-B) of the meshes after MW. Results shown in Table IV ${ }^{7}$ prove a good performance of the MW technique in terms of geometric and fitting quality meeting the requirements of the FEM solver. Fig. 6 shows an illustrative example of the generation of a control-specific mesh.

\section{Visual Classification Protocol}

Scans from the 20 controls, 20 individuals with AD, and the nine simulated atrophy scans were presented in a random order

${ }^{7}$ Note that the registration overlap (RO) measure of the original image is not equal to 1 since the ground-truth was the brain segmentation of the MNI atlas obtained with a semiautomated technique [42], which was slightly different from the brain parcellation given by the MNI labels. 
TABLE V

Atrophy RATING OF THE REAL AND SimUlated DATA

\begin{tabular}{|c||c|c|c|}
\hline & Controls & AD & Simulated AD \\
\hline \hline Average \pm STD & $1.95 \pm 1.42$ & $4.23 \pm 0.98$ & $3.19 \pm 1.36$ \\
\hline \hline
\end{tabular}

to three neurologists independently. The assessors were blinded to any subject-specific information, but were informed of the mean age of the control and $\mathrm{AD}$ groups. They were aware of the categories of scans, but not their distribution. The 3-D volumetric scans were viewed using the MIDAS software [42], and the assessor was able to examine the image in the coronal, axial, or sagittal planes. The neurologists were asked to classify each scan on a five point scale, with a score of one being definite control, and a score of five being definite AD. Sensitivity and specificity for the discrimination of the true $\mathrm{AD}$ cases from the controls were calculated. A kappa score was calculated to assess inter-rater agreement for all scans.

\section{Results}

The mean sensitivity and specificity for visual classification of true $\mathrm{AD}$ from control scans was $90 \%$ and $77 \%$, respectively. These values are similar to other published figures [97]. The inter-rater agreement was moderate, with a kappa score of 0.52 .

The mean score for each scan category is given in Table V. More than half of the simulated atrophy scans were classified as $\mathrm{AD}$ or with a score closer to the AD (5) than to the control (1) group for each assessor. The mean rating for the simulated atrophy scans was somewhere between the definite control score and the definite $\mathrm{AD}$ one.

\section{DISCUSSION}

In this paper, we have used expert clinical knowledge of the rate and spatial distribution of atrophy in dementia to create a phenomenological model of the appearance of atrophy in MRI. The model includes structural remodelling concomitant with atrophy and can include realistic simulation of imaging artefacts.

The mesh generation stage is critical for the good performance of the atrophy simulation methodology. The choice of linear tetrahedral elements allowed the computation of analytical integrands, facilitating the meshing of the structures [68] since a smaller number of elements is required to obtain a smooth surface. Some authors [55], [57] claim that hexahedral or higher order elements are needed to avoid locking effects and to permit the computation of large displacements, when modelling almost incompressible material. Nevertheless, since we worked with displacements, it has been proven that the use of tetrahedral elements does not necessarily involve much error in the displacement estimation [68], [98]. It is obvious that meshes with higher resolution (i.e., a higher amount of elements) would improve the general behaviour of the procedure, but the current resolution has proven to be sufficient to obtain satisfactory results. The introduction of additional labels into the generated volumetric mesh such as the inclusion of hemispheric separation or other anatomical structures will have a strong impact on the ability of the technique to simulate different types of dementia. Furthermore, the use of disease-specific atlases, or those based on elderly individuals, would make the adaptation to a particular patient easier than when using the MNI-ICBM atlas (generated from a young individual).

Thermoelasticity was found to be a convenient way of inducing structural volume change in our application. However, it should be noted that the thermal expansion coefficient in our model does not have a physical meaning beyond introducing an initial stress term that leads to a volume displacement field. This avoids the need for more accurate models of tissue thermal distributions such as the bioheat equation [99]. In consequence, the choice of the elastic properties is not critical in the model presented here, because the boundary conditions enforce a constant global volume, while the relative changes in tissue region volumes are driven by the ratio of thermal coefficients. Taking this into account, we have used a deformation model based on a linear elastic finite-element method. Although it has been demonstrated that brain tissue is a nonlinear viscoelastic material [68], and that nonlinear models are required to simulate large deformations, in this work, we considered structural displacements induced by atrophy that are small in relation to the total brain volume, so that the use of a linear model is justified.

In Section VI, we have presented some results on the simulation of artefacts in the MR scans due to acquisition imperfections. This will allow the evaluation of atrophy measurement techniques, in particular the longitudinal-based ones, with respect to these situations. It is especially relevant to identify the degree of robustness of the registration methods involved in atrophy measurements in the presence of acquisition artefacts, where image analysis is the principle reason for acquiring scans, for instance, as part of large clinical trials. Therefore, a proper understanding of the sensitivity of the algorithms to artefacts will reduce the number of wasted scans and/or of rescans necessary. Furthermore, the increasing use of 3-T MR scanners, which produce images with a higher presence of artefacts, will demand the development of image processing techniques which are tolerant to these artefacts, to allow longitudinal studies with images provided by different acquisition protocols or scanners.

The cross-sectional and longitudinal cases described in Section $\mathrm{V}$ mimic the differences between normal aging controls and AD patients. The proposed methodology could be applied to simulate other types of dementia or degenerative diseases in brain structures, including different topological patterns of atrophy due to frontotemporal dementia in which changes may be very focal in the frontal and temporal lobes changes due to white matter diseases such as vascular disease or multiple sclerosis, changes due to conditions of the CSF such as hydrocephalus and lumbar puncture. The longitudinal example has shown the ability of the procedure to simulate the temporal progression of atrophy changes. This is crucial since the regional distribution of cellular damage due to different pathologies leads to different spatial patterns of atrophy in different types of dementia; many of the current methods fail to separate patients with one type of dementia from those with other disorders. The cross-sectional example is currently limited by the fact that we have just modelled local changes and the topological distribution of the structures is then the same for all simulated scans, which is not the case with real 
scans. Actually, atrophy may impair the spatial registration step applied in the majority of the proposed atrophy measurement methods, and this problem cannot be studied with the current simulations. Future work will combine the atrophy simulation procedure with known global misregistrations or with diffeomorphic transformations obtained either from the mesh warping procedure or by exploiting recent research on the generation of realistic high-dimensional deformations [100].

The geometric and fitting quality of the warped meshes after applying the MW procedure detailed in Section VII-B proved a good performance of the adaptation of the reference atlas-based mesh into the subjects. Nevertheless, an ad hoc refinement procedure, either in terms of remeshing or in terms of element relabelling with information provided by a segmentation procedure applied to the subject image, could considerably improve the appropriateness of the subject-specific meshes.

The simulated scans were not consistently classified as AD, but our results show that even true AD cases may be misidentified in one in 10 cases. This may be due to the large inter-subject variation which exists when these subject groups are compared cross-sectionally. The control group were elderly, and brain atrophy due to normal aging may create overlap when visual classification of MR scans is used. Future work to examine how well the atrophy model performs in simulating longitudinal atrophy would be useful to reduce the effect of this large inter-subject variation. Nevertheless, the visual classification protocol suggests that the model produced AD-simulated scans leading to the same trend in group separation/classification between controls and $\mathrm{AD}$ patients in a cross-sectional study used by expert observers than when using real data. From this observation, we can conclude that an expert may not always be able to discern between the simulated scans and real data, thus implying that our simulation produces results with reasonable degree of realism. In on-going work, we plan to perfom a more detailed quantitative validation of the realism of the simulated images in the future.

In summary, the immediate value of the generated simulated data is to provide gold standard data which can be used to test and validate atrophy measurement techniques commonly used in the study of the neurodegenerative diseases mentioned above. A broader short-term application is the assessment of nonrigid registration and segmentation techniques where it is difficult to validate using anything but the most contrived exemplar datasets. For doing so, we plan to generate a large cohort of brain images with known, physically and clinically plausible, change and make this large database of images freely available to interested groups worldwide through the IXI (Information eXtraction from Images) website, ${ }^{8}$ a U.K. e-Science core-funded project aimed at using image analysis to generate image metadata information about the images and the generic applicability of this technology (grid-enabled image registration and segmentation for drug discovery, medical research, and decision support in healthcare) [101], [102]. This could form the basis of a multisite evaluation in a similar way to previous studies such as the comparison of registration methods published by West $e t a l$. [103].

${ }^{8}$ http://www.ixi.org.uk

\section{CONCLUSION}

We have proposed a method providing valuable ground-truth data for the testing and objective validation of atrophy measurement techniques. In the existing literature, validation has not been done in a satisfactory way since standardized test datasets with known volume changes are not available. Our technique is based on the combination of expert knowledge of clinical changes in brain with finite-element methods, including modelling and solver techniques. Such combination has proven to generate sufficiently realistic simulated MR brain images with local and/or global atrophy, to guide the assessment of atrophy measurement techniques. Future work will focus on the generation and dissemination of several cohorts of data simulating the pattern of changes induced in the human brain by different types of dementia such as fronto-temporal dementia, vascular disease, or multiple sclerosis.

\section{ACKNOWLEDGMENT}

The authors wish to thank C. Frost for his statistical advice, and Dr. J. Kennedy and Dr. J. Rohrer for their contribution to the atrophy rating study.

\section{REFERENCES}

[1] C. Ferri, M. Prince, C. Brayne, H. Brodaty, L. Fratigfioni, M. Ganguli, K. Hall, K. Hasegawa, H. Hendrie, Y. Huang, A. Jorm, C. Mathers, P. Menezes, E. Rimmer, and M. Scazufca, "Global prevalence of dementia: A Delphi consensus study," Lancet, vol. 366, pp. 2112-2117, 2005.

[2] S. Gilman, M. Koller, R. Black, L. Jenkins, S. Griffith, N. Fox, L. Eisner, L. Kirby, M. Boada-Rovira, F. Forette, and J.-M. Orgogozo, "Clinical effects of a $\beta$ immunization (an1792) in patients with AD in an interruped trial," Neurology, vol. 64, pp. 1553-1562, 2005.

[3] N. Fox, R. Scahill, W. Crum, and M. Rossor, "Correlation between rates of brain atrophy and cognitive decline in Alzheimer's disease," Neurology, vol. 52, pp. 1687-1689, 1999.

[4] N. Fox, S. Cousens, R. Scahill, R. Harvey, and M. Rossor, "Using serial registered brain magnetic resonance imaging to measure disease progression in Alzheimer's disease: Power calculations and estimates of sample size to detect treatment effects," Arch. Neurol., vol. 57, pp. 339-344, 2000.

[5] H. Braak and E. Braak, "Neuropathological staging of Alzheimer-related changes," Acta Neuropathologica, vol. 82, no. 4, pp. 239-259, 1991.

[6] N. Fox and P. Freeborough, "Brain atrophy progression measured from registered serial MRI: Validation and application to Alzheimer's disease," J. Magn. Resonan. Imag., vol. 7, pp. 1069-1075, 1997.

[7] D. Chan, N. Fox, R. Scahill, W. Crum, J. Whitwell, G. Leschziner, A. Rossor, J. Stevens, L. Cipolotti, and M. Rossor, "Patterns of temporal lobe atrophy in semantic dementia and Alzheimer's disease," Ann. Neurol., vol. 49, no. 4, pp. 433-442, 2001.

[8] J. O'Brien, S. Paling, R. Barber, E. Williams, C. Ballard, I. McKeith, A. Gholkar, W. Crum, M. Rossor, and N. Fox, "Progressive brain atrophy on serial MRI in dementia with Lewy bodies, AD, and vascular dementia," Neurology, vol. 56, pp. 1386-1388, 2001.

[9] C. Gallon, K. Patterson, K. Graham, M. Lambon-Ralph, G. Williams, N. Antoun, B. Sahakian, and J. Hodges, "Differing patterns of temporal atrophy in Alzheimer's disease and semantic dementia," Neurology, vol. 57, pp. 216-225, 2001.

[10] R. Scahill, C. Frost, R. Jenkins, J. Whitwell, M. Rossor, and N. Fox, "A longitudinal study of brain volume changes in normal aging using serial registered magnetic resonance imaging," Arch. Neurol., vol. 60, pp. 989-994, 2003.

[11] S. Resnick, D. Pham, M. Kraut, A. Zonderman, and C. Davatzikos, "Longitudinal magnetic resonance imaging studies of older adults: A shrinking brain," J. Neurosci., vol. 23, no. 8, pp. 3295-3301, 2003.

[12] R. Walters, N. Fox, J. Schott, W. Crum, J. Stevens, M. Rossor, and D. Thomas, "Transient ischaemic attacks are associated with increased rates of global cerebral atrophy,' J. Neurol. Neurosurg. Psychiatry, vol. 74 , pp. 213-216, 2003. 
[13] E. Sullivan, A. Pfefferbaum, E. Adalsteinsson, G. Swan, and D. Carmelli, "Differential rates of regional brain change in callosal and ventricular size: A 4-year longitudinal MRI study of elderly men," Cereb. Cortex, vol. 12, no. 4, pp. 438-445, 2002.

[14] P. Thompson, K. Hayashi, G. de Zubicaray, A. Janke, S. Rose, J. Semple, M. Hong, D. Herman, D. Gravano, D. Doddrell, and A. Toga, "Mapping hippocampal and ventricular change in Alzheimer's disease," NeuroImage, vol. 22, no. 4, pp. 1754-1766, 2004.

[15] B. Dickerson and R. Sperling, "Neuroimaging biomarkers for clinical trials of disease-modifying therapies in Alzheimer's disease," J. Amer. Soc. Exper. NeuroTherapeutics, vol. 2, pp. 348-360, 2005.

[16] J. Growdon, D. Selkoe, A. Roses, J. Trojanowski, P. Davies, S. Appel, S. Gilman, T. Radebaugh, and Z. Khachaturian, "Committee WGA: Consensus report of the working group on biological markers of Alzheimer's disease. Ronald and Nancy Reagan Institute of the Alzheimer's Association and National Institute on Aging Working Group on Biological Markers of Alzheimer's Disease," Neurobiol. Aging, vol. 19, pp. 109-116, 1998.

[17] D. Mungas, B. Reed, W. Jagust, C. DeCarli, W. Mack, J. Kramer, M. Weiner, N. Schuff, and H. Chui, "Volumetric MRI predicts rate of cognitive decline related to AD and cerebrovascular disease," Neurology, vol. 56, pp. 1386-1388, 2002.

[18] J. Ashburner, J. Csenansky, C. Davatzikos, N. Fox, G. Frisoni, and P. Thompson, "Computer-assisted imaging to assess brain structure in healthy and diseased brains," Lancet Neurol., vol. 2, pp. 79-88, 2003.

[19] U. Grenander and M. Miller, "Computational anatomy: An emerging discipline," Quart. Appl. Math., vol. 4, pp. 617-694, 1998.

[20] J. Ashburner and K. Friston, "Voxel based morphometry-the methods," NeuroImage, vol. 11, pp. 805-821, 2000.

[21] C. Davatzikos, A. Gene, D. Xu, and S. Resnick, "Voxel-based morphometry using the RAVENS maps: Methods and validation using simulated longitudinal atrophy," NeuroImage, vol. 14, no. 6, pp. 1361-1369, 2001.

[22] P. Thompson, M. Mega, R. Woods, C. Zoumalan, C. Lindshield, R. Blanton, J. Moussai, C. Homes, J. Cummings, and A. Toga, "Cortical change in Alzheimer's disease detected with a disease-specific population based brain atlas," Cereb. Cortex, vol. 11, no. 1, pp. 1-16, 2001.

[23] P. Thompson, K. Hayashi, E. Sowell, N. GogTay, J. Giedd, J. Rapoport, G. de Zubicaray, A. Janke, S. Rose, J. Semple, D. Doddrell, Y. Wang, T. van Erp, T. Cannon, and A. Toga, "Mapping cortical change in Alzheimer's disease, brain development, and schizophrenia," NeuroImage, vol. 23, pp. S2-S18, 2004.

[24] B. Fischl and A. Dale, "Measuring the thickness of the human cerebral cortex from magnetic resonance images," Proc. Nat. Acad. Sci. USA, vol. 97, no. 20, pp. 11050-11055, 2000.

[25] J. Lerch, J. Pruessner, A. Zijdenbos, H. Hample, S. Teipel, and A. Evans, "Focal decline of cortical thickness in Alzheimer's disease identified by computational neuroanatomy," Cereb. Cortex, vol. 15, pp. 995-1001, 2005.

[26] J. Csernansky, S. Joshi, L. Wang, J. Haller, M. Gado, J. Miller, U. Grenander, and M. Miller, "Hippocampal morphometry in schizophrenia via high dimensional brain mapping," Nat. Acad. Sci. USA, vol. 95, pp. 11406-11411, 1998.

[27] Z. Lao, D. Shen, Z. Xue, B. Karacali, S. Resnick, and C. Davatzikos, "Morphological classification of brains via high-dimensional shape transformations and machine learning methods," NeuroImage, vol. 21, no. 1, pp. 46-57, 2004.

[28] S. Smith, Y. Zhang, M. Jenkinson, J. Shen, P. Matthews, A. Federico, and N. D. Stefano, "Accurate, robust, and automated longitudinal and cross-sectional brain change analysis," NeuroImage, vol. 17, pp. 479-489, 2002.

[29] P. Freeboiough and N. Fox, "The boundary shift integral: An accurate and robust measure of cerebral volume changes from registered repeat MRI," IEEE Trans. Med. Imag., vol. 16, no. 5, pp. 623-629, Oct. 1997.

[30] S. Smith, N. D. Stefano, M. Jenkinson, and P. Matthews, "Normalized accurate measurement of longitudinal brain change," J. Comput. Assist. Tomogr., vol. 25, no. 3, pp. 466-475, 2001.

[31] D. Wang and D. Doddrell, "MR image-based measurement of rates of change in volumes of brain structures. Part I: Method and validation," J. Magn. Reson. Imag., vol. 20, pp. 27-40, 2002.

[32] K. Chen, E. Reiman, G. Alexander, D. Bandy, R. Renaut, W. Crum, N. Fox, and M. Rossor, "An automated algorithm for the computation of brain volume change from sequential MRIs using an iterative principal component analysis and its evaluation for the assessment of whole-brain atrophy rates in patients with probable Alzheimer's disease," NeuroImage, vol. 22, pp. 134-143, 2004.
[33] P. Freeborough, N. Fox, and R. Kitney, "Modeling brain deformations in Alzheimer's disease by fluid registration of serial 3-D MR images," J. Comput. Assist. Tomogr., vol. 22, pp. 838-843, 1998.

[34] G. Chételat, B. Landeau, F. Eustache, F. Mézenge, F. Viader, V. de la Sayette, B. Desgranges, and J.-C. Baron, "Using voxel-based morphometry to map the structural changes associated with rapid conversion in MCI: A longitudinal MRI study," NeuroImage, vol. 27, pp. 934-946, 2005.

[35] R. Scahill, J. Schott, J. Stevens, M. Rossor, and N. Fox, "Mapping the evolution of regional atrophy in Alzheimer's disease: Unbiased analysis of fluid-registered serial MRI," Nat. Acad. Sci. USA, vol. 99, no. 7, pp. 4703-4707, 2002.

[36] P. Thompson, K. Hayashi, G. de Zubicaray, A. Janke, S. Rose, J. Semple, D. Herman, M. Hong, S. Dittmer, D. Doddrell, and A. Toga, "Dynamics of gray matter loss in Alzheimer's disease," J. Neurosci., vol. 23, no. 3, pp. 994-1005, 2003.

[37] W. Crum, R. Scahill, and N. Fox, "Automated hippocampal segmentation by regional fluid registration of serial MRI: Validation and application in Alzheimer's disease," NeuroImage, vol. 13, no. 5, pp. 847-855, 2001.

[38] Z. Kue, D. Shen, and C. Davatzikos, "CLASSIC: consistent longitudinal alignment and segmentation for serial image computing," Neurolmage, vol. 21, no. 5, pp. 46-57, 2005.

[39] B. Karacali and C. Davatzikos, "Simulation of tissue atrophy using a topology preserving transformatio model," IEEE Trans. Med. Imag., vol. 25, no. 5, pp. 649-652, May 2006.

[40] J. Schnabel, C. Tanner, A. Castellano-Smith, A. Degenhard, M. Leach, D. Hose, D. Hill, and D. Hawkes, "Validation of nonrigid image registration using finite-element methods: Application to breast MR images," IEEE Trans. Med. Imag., vol. 22, no. 2, pp. 238-247, Feb. 2003.

[41] D. Collins, A. Zijdenbos, V. Kollokian, J. Sled, N. Kabani, C. Holmes, and A. Evans, "Design and construction of a realistic digital brain phantom," IEEE Trans. Med. Imag., vol. 17, no. 3, pp. 463-468, Jun. 1998.

[42] P. Freeborough, N. Fox, and R. Kitney, "Interactive algorithms for the segmentation and quantitation of 3-D MRI brain scans," Comput. Meth. Programs Biomed., vol. 53, pp. 15-25, 1997.

[43] W. Lorensen and H. Cline, "Marching cube, a high resolution 3-D surface reconstruction algorithm," in Proc. Int. Conf. Comput. Graphics Interactive Techn. (SIGGRAPH'87), 1987, vol. 21, pp. 163-169.

[44] J. Schoberl, "NETGEN-An advancing front 2D/3-D-mesh generator based on abstract rules," Comput. Visualizat. Sci., vol. 1, pp. 41-52, 1997.

[45] J. J. Shewchuk, "What is a good linear element? Interpolation, conditioning and quality measures," in Proc. 11th Int. Mesh. Roundtable, 2002, pp. 115-126.

[46] M. Sermesant, C. Forest, X. Pennec, H. Delingette, and N. Ayache, "Deformable biomechanical models: Application to 4D cardiac image analysis," Med. Image Analy., vol. 7, pp. 475-488, 2003.

[47] D. Cash, M. Miga, T. Sinha, R. Galloway, and W. Chapman, "Compensating for intra-operative soft tissue deformations using incomplete surface data and finite elements," IEEE Trans. Med. Imag., vol. 24, no. 11, pp. 1479-1491, Nov. 2005.

[48] K. Brock, S. Hollister, L. Dawson, and J. Balter, "Technical note: Creating a four-dimensional model of the liver using finite element analysis," Med. Phys., vol. 29, no. 7, pp. 1403-1405, 2002.

[49] K. Miller, "Constitutive modelling of abdominal organs," J. Biomech., vol. 33, pp. 367-373, 2000.

[50] M. Sermesant, K. Rhode, G. Sanchez-Ortiz, O. Camara, R. Andriantsimiavona, S. Hegde, D. Rueckert, P. Lambiase, C. Bucknall, E. Rosenthal, H. Delingette, D. Hill, N. Ayache, and R. Razavi, "Simulation of cardiac pathologies using an electromechanical biventricular model and XMR interventional imaging," Med. Image Anal., vol. 9, pp. 467-480, 2005.

[51] A. Samani, J. Bishop, M. Yaffe, and D. Plewes, "Biomechanical 3-D finite-element modeling of the human breast using MRI data," IEEE Trans. Med. Imag., vol. 20, no. 4, pp. 271-279, Apr. 2000.

[52] D. Plewes, J. Bishop, A. Samani, and J. Sciaretta, "Visualization and quantification of breast cancer biomechanical properties with magnetic resonance elastography," Phys. Med. Biol., vol. 45, pp. 1649-1664, 2000.

[53] F. Azar, D. Metaxas, and M. Schnall, "A deformable finite element model of the breast for predicting mechanical deformations under external perturbations," Academic Radiol., vol. 8, pp. 965-975, 2001.

[54] M. Miga, "A new approach to elastography using mutual information and finite elements," Phys. Med. Biol., vol. 48, pp. 467-480, 2003. 
[55] O. Skrinjar, C. Studholme, A. Nabavi, and J. Duncan, "Steps toward a stereo-camera-guided biomechanical model for brain shift compensation," in Proc. Conf. Inf. Process. Med. Imag. (IPMI'OI), 2001, vol. 2082, pp. 183-189.

[56] M. Ferrant, A. Nabavi, B. Macq, F. Jolesz, R. Kikinis, and S. Warfield, "Registration of 3-D intraoperative MR images of the brain using a finite element biomechanical model," IEEE Trans. Med. Imag., vol. 20, no. 12, pp. 1384-1397, Dec. 2001.

[57] K. Miller, "Constitutive model of brain tissue suitable for finite element analysis of surgical procedures," J. Biomech., vol. 32, pp. 531-537, 1999.

[58] L. Platenik, M. Miga, D. Roberts, K. Lunn, F. Kennedy, A. Hartov, and K. Paulsen, "In vivo quantification of retraction deformation modeling for updated image-guidance during neurosurgery," IEEE Trans. Med. Imag., vol. 49, no. 8, pp. 823-835, Aug. 2002.

[59] A. Castellano-Smith, T. Hartkens, J. Schnabel, R. Hose, H. Liu, W. Hall, C. Truwit, D. Hawkes, and D. Hill, "Constructing patient specific models for correcting intraoperative brain deformation," in Proc. Int. Conf. Med. Image Comput. Comput.-Assisted Intervention (MICCA'01), 2001, pp. 1091-1098.

[60] O. Clatz, H. Delingette, I.-F. Talos, A. Golby, R. Kikinis, F. Jolesz, N. Ayache, and S. Warfield, "Robust nonrigid registration to capture brain shift from intraoperative MRI," IEEE Trans. Med. Imag., vol. 17, no. 11, pp. 463-468, Nov. 2005.

[61] A. Hagemann, K. Rohr, H. Stiehl, U. Spetzger, and J. Gilsbach, "Biomechanical modeling of the human head for physically based, nonrigid image registration," IEEE Trans. Med. Imag., vol. 18, no. 10, pp. 875-884, Oct. 1999.

[62] O. Clatz, M. Sermesant, P.-Y. Bondiau, H. Delingette, S. Warfield, G. Malandain, and N. Ayache, "Realistic simulation of the 3-D growth of brain tumors in MR images coupling diffusion with biomechanical deformation," IEEE Trans. Med. Imag., vol. 24, no. 10, pp. 1334-1346, Oct. 2005

[63] K. Miller and K. Chinzei, "Mechanical properties of brain tissue in tension," J. Biomech., vol. 35, pp. 483-490, 2002.

[64] M. Schweiger and S. Arridge, "Image reconstruction in optical tomography using local basis functions," J. Electron. Imag., vol. 12, pp. 583-593, 2003.

[65] O. Zienkiewicz and R. Taylor, The Finite Element Method, 4th ed. London, U.K.: McGraw-Hill, 1987.

[66] S. Kruse, M. Dresner, P. Rossman, J. Felmlee, C. Jack, and R. Ehman, "Palpation of the brain using magnetic resonance elastography," in Proc. 7th Ann. Meeting ISMRM, 1999, vol. 1, pp. 281-297.

[67] P. McCracken, A. Manduca, J. Felmlee, and R. Ehman, "Mechanical transient-based magnetic resonance elastography," Magn. Reson. Med., vol. 53, no. 20, pp. 628-639, 2005.

[68] T. Carter, M. Sermesant, D. Cash, D. Barratt, C. Tanner, and D. Hawkes, "Application of soft tissue modelling to image-guided surgery," Med. Eng. Phys., vol. 27, pp. 893-909, 2005.

[69] A. J. Davies, The Finite Element Method: A First Approach. London, U.K.: Oxford Univ. Press, 1980.

[70] R. W. Hamming, Digital Filters, ser. Signal Processing Series, 3rd ed. New York: Prentice-Hall, 1989.

[71] S. Lee, G. Wolberg, and S. Y. Shin, "Scattered data interpolation with multilevel B-splines," IEEE Trans. Visualizat. Comput. Graphics, vol. 3, no. 3, pp. 228-244, Jul.-Sep. 1997.

[72] G. V. Hoesen, "Anatomy of the medial temporal lobe," J. Magn. Reson. Imag., vol. 13, pp. 1047-1055, 1995.

[73] A. C. M. J. de Leon, S. De Santi, M. Bobinski, A. George, H. Wisniewski, H. Rusinek, R. Carroll, and L. S. Louis, "Contribution of structural neuroimaging to the early diagnosis of Alzheimer's disease," Int. Psychogeriatrucs, vol. 9, no. Suppl 1, pp. 183-190, 1997.

[74] E. Mori, Y. Yoneda, H. Yamashita, N. Hirono, M. Ikeda, and A. Yamadori, "Medial temporal structures relate to memory impairment in Alzheimer's disease: An MRI volumetric study," J. Neurol. Neurosurg. Psychiatry, vol. 63, pp. 214-221, 1997.

[75] J. Krasuski, G. Alexander, B. Horwitz, E. Daly, D. Murphy, S. Rapoport, and M. Schapiro, "Volumes of medial temporal lobe structures in patients with Alzheimer's disease and mild cognitive impairment (and in healthy controls)," Biol. Psychiatry, vol. 43, pp. 60-68, 1998.

[76] L. DeToledo-Morrell, I. Goncharova, B. Dickerson, R. Wilson, and D. Bennett, "From healthy aging to early Alzheimer's disease: In vivo detection of entorhinal cortex atrophy," Ann. New York Acad. Sci., vol. 911, pp. 240-253, 2000.

[77] A. Janke, G. Zubicaray, S. Rose, M. Griffin, J. Chalk, and G. Galloway, " $4 \mathrm{~d}$ deformation modeling of cortical disease progression in Alzheimer's dementia," Magn. Reson. Med., vol. 53, no. 20, pp. 628-639, 2000.
[78] P. Thompson, T. Cannon, K. Narr, T. van Erp, V. Poutanen, M. Huttunen, J. Lonnqvist, C. Standertskjold-Nordenstam, J. Kaprio, M Khaledy, R. Dail, C. Zoumalan, and A. Toga, "Genetic influences on brain structure," Nature Neurosci., vol. 4, no. 12, pp. 1253-1258, 2001.

[79] A. Du, N. Schuff, X. Zhu, W. Jagust, B. Miller, B. Reed, J. Kramer D. Mungas, K. Yaffe, H. Chui, and M. Weiner, "Atrophy rates of entorhinal cortex in AD and normal aging," Neurology, vol. 60, pp. 481-486, 2003.

[80] K. Kantarci and C. Jack, Jr., "Quantitative magnetic resonance techniques as surrogate markers of Alzheimer's disease," J. Amer. Soc. Exper. NeuroTherapeutics, vol. 1, pp. 196-205, 2004.

[81] H. Braak and E. Braak, "Evolution of the neuropathology of Alzheimer disease," Acta Neurologica Scandinavica. Suppl., vol. 165, pp. 3-12, 1996.

[82] K. Juottonen, M. Laakso, R. Insausti, M. Lehtovirta, A. Pitkanen, K. Partanen, and H. Soininen, "Volumes of the entorhinal and perirhinal cortices in Alzheimer disease," Neurobiol. Aging, vol. 18, pp. 1-11, 1991.

[83] C. Jack, Jr., M. Shiung, J. Gunter, P. O'Brien, S. Weigand, D. Knopman, B. Boeve, R. Ivnik, G. Smith, R. Cha, E. Tangalos, and R. Petersen, "Comparison of different MRI brain atrophy rate measures with clinical disease progression in AD," Neurology, vol. 62, pp. 591-600, 2004

[84] N. Fox, R. Black, S. Gilman, M. Rossor, S. Griffith, L. Jenkins, and M. Roller, "Effects of A $\beta$ immunization (AN1792) on MRI measures of cerebral volume in Alzheimer's disease," Neurology, vol. 52, pp. 1687-1689, 1999.

[85] H. Braak and E. Braak, "Staging of Alzheimer's disease-related neurofibrillary changes," Neurobiol. Aging, vol. 16, pp. 271-278, 1995.

[86] G. Preboske, J. Gunter, C. Ward, and C. Jack, Jr., "Common MRI acquisition non-idealities significantly impact the output of the boundary shift integral method of measuring brain atrophy on serial MRI," NeuroImage, to be published.

[87] J. Schott, S. Price, C. Frost, J. Whitwell, M. Rossor, and N. Fox, "Measuring atrophy in Alzheimer disease: A serial MRI study over 6 and 12 months," Neurology, vol. 52, pp. 1687-1689, 1999.

[88] D. Holdsworth, C. Norley, R. Frayne, D. Steinman, and B. Rutt, "Characterization of common carotid artery blood-flow waveforms in normal human subjects," Physiological Meas., vol. 20, pp. 219-240, 1999.

[89] G. McKhann, D. Drachman, M. Folstein, R. Katzman, D. Price, and E. Stadlan, "Clinical diagnosis of Alzheimer's disease: report of the NINCDS-ADRDA work group under the auspices of department of health and human services task force on alzheimer's disease," $\mathrm{Neu}$ rology, vol. 34, pp. 939-944, 1984.

[90] N. Fox, H. Warrington, P. Freeborough, P. Hartikainen, A. Kennedy, J. Stevens, and M. Rossor, "Presymptomatic hippocampal atrophy in Alzheimer's disease. A longitudinal MRI study," Brain, vol. 119, pp. 2001-2007, 1996.

[91] A. Castellano-Smith, T. Hartkens, J. Schnabel, D. Hose, H. Liu, W. Hall, C. Truwit, D. Hawkes, and D. Hill, "A registration based mesh construction technique for finite element models of brains," in Proceedings of SPIE Medical Imaging. Bellingham, WA: SPIE, 2002, vol. 4683, pp. 538-549.

[92] O. Camara, W. Crum, J. Schnabel, E. Lewis, M. Schweiger, D. Hill, and N. Fox, "Assessing the quality of mesh-warping in normal and abnormal neuroanatomy," in Proc. Conf. Med. Image Understand. Anal. (MIUA'05), 2005, pp. 79-82.

[93] B. Couieau, Y. Payan, and S. Lavallée, "The mesh-matching algorithm: An automatic 3-D mesh generator for finite element structures,' J. Biomech., vol. 33, pp. 1005-1009, 2000.

[94] C. Studholme, D. Hill, and D. Hawkes, "An overlap invariant entropy measure of 3-D medical image alignment," Pattern Recognit., vol. 32, pp. 71-86, 1999.

[95] W. Crum, C. Tanner, and D. Hawkes, "Anisotropic multi-scale fluid registration: Evaluation in magnetic resonance breast imaging," Phys. Med. Biol., vol. 50, pp. 5153-5174, 2005.

[96] A. K. Jain and R. C. Dubes, Algorithms for Clustering Data. Englewood Cliffs, NJ: Prentice-Hall, 1988.

[97] P. Scheltens, N. Fox, F. Barkhof, and C. De Carli, "Structural magnetic resonance imaging in the practical assessment of dementia: Beyond exclusion," Lancet Neurol., vol. 1, pp. 13-21, 2002.

[98] K. Bathe, Finite Element Procedures. Englewood Cliffs, NJ: Prentice-Hall, 1996.

[99] J. Durkee and P. Antich, "Exact solutions to the multi-region timedependent bioheat equation with transient heat sources and boundary conditions," Phys. Med. Biol., vol. 36, no. 3, pp. 345-368, 1991. 
[100] Z. Xue, D. Shen, B. Karacali, and C. Davatzikos, "Statistical representation and simulation of high-dimensional deformations: Application to synthesizing brain deformations," in Proc. Int. Conf. Med. Image Comput. Comput-Assisted Intervention (MICCAI'05), 2005, pp. 500-508.

[101] A. Rowland, T. Hartkens, M. Bums, J. Hajnal, D. Rueckert, and D. Hill, "A grid enabled medical image database," presented at the UK e-Science All Hands Meeting, Nottingham,, U.K., Sep. 2004.

[102] K. Leung, M. Holden, R. Heckmann, N. Saeed, K. Brooks, J. Buckton, K. Changani, D. Reid, D. Rueckert, J. Hajnal, and D. Hill, "Use of data provenance and the grid in medical image analysis and drug discovery-an $1 \times 1$ exemplar," presented at the UK e-Science All Hands Meeting, Nottingham,, U.K., Sep. 2004.
[103] J. West, J. Fitzpatrick, M. Wang, B. Dawant, C. Maurer, Jr., R. Kessler, R. Maciunas, C. Barillot, D. Lemoine, A. Collignon, F. Maes, P. Suetens, D. Vandermeulen, P. van den Elsen, P. Hemler, S. Napel, T. Sumanaweera, B. Harkness, D. Hill, C. Studholme, G. Malandain, X. Pennec, M. Noz, G. Maguire, Jr., M. Pollack, C. Pellizari, R. Robb, D. Hanson, and R. Woods, "Comparison and evaluation of retrospective intermodality brain image registration techniques," in SPIE Conf. Med. Imag.: Image Process., 1996, vol. 2710, pp. 332-347. 